\title{
Novel clinical and therapeutic aspects in autoimmunity
}

\author{
Howard Amital $^{1}$ - Abdulla Watad ${ }^{1} \cdot$ Zoltán Szekanecz $^{2}$
}

Published online: 24 September 2016

(C) Springer Science+Business Media New York 2016

The Autoimmunity Congress has become a tradition and an important biannual event in the world of autoimmunity.

The following issue of Immunological Research is dedicated to the important contributions that were presented during the recent Autoimmunity Congress that was held in Leipzig, Germany, on April 6-10, 2016, attended by 2210 participants. We will go through the essentials of these publications referring to novel clinical and therapeutic aspects in the following report.

One-third of patients with rheumatoid arthritis treated with adalimumab may develop anti-adalimumab antibodies. These antibodies are associated with reduced drug levels, loss of drug efficacy, clinical non-responsiveness and an increased risk of adverse effects. Monitoring drug and anti-drug antibody levels appears to be a putative strategy for optimal and cost-effective intervention [1, 2].

Ogrič et al. [3] reviewed the progress and the pitfalls of modern bioassays detecting anti-adalimumab antibodies. It is evident that successful detection of anti-ADL antibodies depends on selection of a proper assay, its capacity to break down potential immune complexes and to detect anti-ADL antibodies. This review presents the diverse methodologies available nowadays with their advantages and drawbacks.

Henoch-Schönlein purpura (HSP) or immunoglobulin A ( $\operatorname{Ig} \mathrm{A})$ vasculitis is an immune complex vasculitis affecting

Howard Amital

howard.amital@sheba.health.gov.il

1 Department of Medicine 'B', Sackler Faculty of Medicine, Zabludowicz Center for Autoimmune Diseases, Sheba Medical Center, Tel-Aviv University, Tel-Hashomer, Israel

2 Department of Rheumatology, Department of Medicine, University of Debrecen, Medical and Health Sciences Center, Debrecen, Hungary small vessels with dominant IgA deposits [4]. It is characterized by a tetrad of palpable purpuric rash, arthralgia/arthritis, gastrointestinal symptoms (i.e., abdominal pain, gastrointestinal bleeding) and renal disease. Fenoglio et al. [5] summarized their clinical experience with rituximab therapy for HSP. There is evidence that glucocorticoids may reduce the severity and enhance resolution of extrarenal symptoms, especially arthritis, abdominal pain and swelling, yet we do not have solid data regarding glucocorticoid efficacy in patients with nephritis. The authors describe their promising experience with rituximab in a small series of adult HSP with biopsy-proven aggressive glomerulonephritis. All patients achieved a complete renal remission.

Concurrent association of idiopathic thrombocytopenic purpura (ITP) and peripheral neuropathy is a rare condition. There are only a few case reports published concerning peripheral neuropathy with ITP. In their report Katchan et al. [6] describe a case study of a 47-year-old woman who was diagnosed with a two-year history of idiopathic thrombocytopenic purpura and who later developed a chronic combined inflammatory demyelinating polyneuropathy. While high-dose corticosteroid therapy yielded no benefit, the patient showed a significant improvement of platelet count and of the neuropathic symptoms following therapy intravenous immunoglobulin.

Systemic lupus erythematosus (SLE) is an autoimmune disease with multiple organ involvement. It is characterized by abundant autoantibodies that form immune complex with autoantigens and deposit in organs and cause tissue damage by inducing inflammation. The pathogenesis of SLE has been intensively studied but remains unclear. B and $\mathrm{T}$ lymphocyte abnormalities, dysregulation of apoptosis, defects in the clearance of apoptotic materials and various genetic and epigenetic factors are believed to contribute to the initiation and development of SLE [7]. 
Marinho et al. [8] alluded to the linkage between active SLE with low regulatory T cells levels. This group assessed whether vitamin D supplementation modified the FoxP3 expression and IL-17A production of T cells in 24 patients with SLE. Indeed, the FoxP3 $3^{+} / \mathrm{IL}-17 \mathrm{~A}$ ratio in SLE patients after 6 months of vitamin D supplementation was higher than that at baseline $(p<0.001)$ in parallel with the increment of serum $25(\mathrm{OH}) \mathrm{D}$ levels throughout this period.

Glaucomatous optic neuropathy is the most commonly acquired optic neuropathy encountered in clinical practice and the second leading cause of blindness; yet its autoimmune pathogenesis is unknown. In her elegant review Rizzo et al. [9] dwelled into aspects that are usually not mentioned. Another uncommon clinical presentation is autoimmune mastitis with coexistent Sjögren's syndrome. Goulabchand et al. [10] investigated mammary involvement in patients with Sjögren's syndrome and compared mammary histological findings with minor salivary gland involvement. They reviewed the charts of patients with Sjögren's syndrome from the Montpellier University Hospital, between 2000 and 2015, where minor salivary gland and mammary tissues were available. Of the 16 patients detected mammary biopsy was conducted because of breast symptoms in 6 patients and following imaging screening strategies for breast cancer in 10 patients. Nine patients exhibited an inflammatory breast pattern (lymphocytic infiltrates or duct ectasia), close to minor salivary gland histological findings. Immunohistochemical staining in five patients revealed $\mathrm{B}$ and $\mathrm{T}$ cell infiltrates within the breast tissue, with a higher proportion of T CD4+ cells, but no IgG4-secreting plasma cells were found.

Migraine is the most frequent central nervous system (CNS) complaint in primary anti-phospholipid syndrome (APS) patients, and there is no association with a specific anti-phospholipid antibodies (aPL) [11]. Hassan et al. [12] reviewed the medical literature regarding the diagnosis and therapeutic significance of migraines and headaches in the anti-phospholipid syndrome (APS). They found that migraine is the most commonly reported type of headache in APS. Several studies reported higher levels of antiphospholipid antibodies in primary and secondary APS migraineurs. They concluded that an anticoagulation trial is warranted in APS patients with migraines since many cases of severe, refractory migraine resolved once anticoagulation therapy was commenced.

Type 1 diabetes (T1D) is a chronic autoimmune disease characterized by the loss of insulin-producing pancreatic $\beta$ cells. The pathogenesis of T1D is complex and multifactorial and involves a genetic susceptibility that predisposes to abnormal immune responses in the presence of ill-defined environmental insults to the pancreatic islets [13]. In their review Gianchecchi et al. [14] presented experimental data from animal models and human studies supporting the role played by the gut microbiome (GM) in the pathogenesis of T1D. The authors mention the differences in the intestinal microbiome of subjects affected by prediabetes or overt disease and the delicate microbiota-host homeostasis as well as the factors leading to its disturbance.

The relationship between infectious agents, such as viruses, and autoimmunity may be crucial [15]. Therefore, Gendelman et al. [16] assessed the prevalence of hepatitis $\mathrm{B}$ virus (HBV) carrier state among patients with systemic lupus erythematosus (SLE), in comparison with age- and sex-matched controls. HBV carriage was determined according to clinical records in the large Clalit Health Services database. Altogether 5018 SLE patients and 25,090 matched non-SLE controls were included in the study. HBV positivity was significantly more common among SLE patients $(0.9 \%)$ compared to controls $(0.56 \%$, $p<0.001)$. In SLE patients, HBV carrier state was associated with a lower socioeconomic status. In a multivariate logistic regression analysis, SLE was significantly associated with $\mathrm{HBV}$ with an odds ratio (OR) of $1.83(95 \%$ $\mathrm{CI}=1.291-2.590)$.

Many attempts are made in order to refine the way we characterize autoimmune disorders. This concept is in particular pursued in the field of inflammatory myopathies. Shovman et al. [17] elaborated on the diagnostic value of anti-HMGCR autoantibodies in necrotizing myopathy following statin exposure. In contrast to dermatomyositis this syndrome is characterized by a typical myopathic phenotype including progressive muscle weakness, arthralgia, myalgia, dysphagia and Raynaud's phenomenon, while multisystem manifestations are scarce. In their study sera samples of 13 statin-exposed patients who were suspected of having necrotizing myopathy following statin exposure were analyzed; 38 patients with different inflammatory and autoimmune rheumatic diseases and 29 healthy subjects were collected. In addition, they also compared the accuracy of two assays: a new chemiluminescence QUANTA Flash HMGCR kit utilizing BIO-FLASH System and the QUANTA Lite ${ }^{\circledR}$ HMGCR ELISA kit. Twelve out of 13 samples from patients with suspicion of necrotizing myopathy following statin exposure were found to be positive for the anti-HMGCR antibodies by both assays. Only one of the 13 samples that were found positive by ELISA was negative by CIA. A very good qualitative correlation (kappa $=0.95 ; 95 \%$ CI, 0.85-1.0) and quantitative agreement (Spearman's rho $0.87 ; P$ value $<0.0001 ; 95 \%$ CI, $0.62-0.96)$ were found between these two assays. All samples from healthy subjects and from the disease-control patient cohort were negative for anti-HMGCR antibodies. In comparison with ELISA results, the CIA assay exhibited high sensitivity and specificity values of 92.3 and $100 \%$, respectively. These tests were found to be highly distinctive for the anti-HMGCR antibodies. 
Adjuvants have recently been implicated in the new syndrome named "autoimmune/inflammatory syndrome induced by adjuvants" (ASIA), which describes an umbrella of clinical conditions including post-vaccination adverse reactions [18]. As an interesting complication of vaccination, Blitshteyn et al. [19] describe the occurrence of postural tachycardia syndrome (POTS) associated with the production of anti-NMDA receptor antibodies after human papillomavirus (HPV) vaccination. They describe a case of an 18-year-old female who developed POTS with positive serum anti-NMDA receptor antibodies but no evidence of encephalitis after HPV vaccination. AntiNMDA receptor antibodies have been most commonly associated with autoimmune encephalitis presenting with acute neurological and psychiatric symptoms. In the present case, symptoms improved significantly after immunosuppression but relapsed after stopping therapy suggesting an autoimmune etiology for POTS after vaccination.

Even local oily vitamin injections can trigger "ASIA," the autoimmune/inflammatory syndrome induced by adjuvants. Balbi et al. [20] reported two cases of patients that rapidly developed clinical features resembling systemic sclerosis after multiple intramuscular local injections of a substance recommended by a non-medical professional called ADE. ADE is an oily vitamin complex for veterinary use; it was used in these cases for cosmetic muscular definition and enhancement purpose. Further investigations may lead us to the better understanding of the pathophysiology of this condition.

In the last few years, a number of studies have been published on a condition characterized by Raynaud's phenomenon (RP) associated with systemic sclerosis (SSc) marker autoantibodies and/or scleroderma-type capillaroscopic abnormalities and referred to as very early/early SSc [21]. It is very important to understand how undifferentiated connective tissue disease (UCTD) transition occurs into a well-defined autoimmune disease. Antinuclear antibody (ANA) positivity suggests a higher risk of transition. An anti-dense fine speckled (DFS) immunofluorescence pattern can be observed when using an ANA test on HEp-2 cells and is due to the presence of antibodies to the nuclear DFS70 antigen. Anti-DFS70 antibodies may be present in 8-40\% of UCTD cases. Infantino et al. [22] described two patients with UCTD that are ANA and anti-DFS70 antibodies.

Chronic comorbidities are important factors determining the prognosis and survival of patients with various autoimmune disorders. The increased mortality and morbidity among patients with rheumatic diseases is mainly due to the high prevalence of ischemic heart disease [23]. Arrhythmias may also influence disease outcome. For example, QTc time prolongation on ECG has been associated with consequent arrhythmias. Chronic systemic inflammation and pro-inflammatory cytokines including TNF- $\alpha$ and IL- 6 play an important role in the prolongation of the QT interval. In addition, long QTc has been related to a high disease activity, elevated hsCRP and increased all-cause mortality in RA. Also, biologics may affect QTc and thus arrhythymias. Lazzerini et al. [24] address the importance of arrhythmias associated with chronic arthritis and the controversial effects of anti-rheumatic therapies. Anti-TNF- $\alpha$ agents and tocilizumab have shown beneficial effects on the QT interval, as well as on cardiac autonomic dysfunction. On the other hand, there have been a few reported cases of RA or inflammatory bowel disease where anti-TNF- $\alpha$ biologics resulted in supraventricular or ventricular tachycardia, bradyarrhythmia or conduction disturbances. In addition, rituximab, mostly in lymphoma patients, also caused arrhythmias in some individuals. There has been one report of sudden death. Most of the patients had no preexisting heart conditions. Anti-TNF- $\alpha$ biologics and rituximab may cause arrhythmias by leading to left ventricular dysfunction, vasospasm, coronary plaque destabilization or complement-mediated myocardial insult and thus further aggravate the pro-arrhytmogenic substrate.

In conclusion during the Leipzig Autoimmunity Congress a wide range of topics were covered. We are sure that the next congress to be held in Lisbon in 2018 will be of even greater success and interest.

\section{References}

1. Meroni PL, Valentini G, Ayala F, Cattaneo A, Valesini G. New strategies to address the pharmacodynamics and pharmacokinetics of tumor necrosis factor (TNF) inhibitors: a systematic analysis. Autoimmun Rev. 2015;14(9):812-29.

2. Deehan M, Garcês S, Kramer D, Baker MP, Rat D, Roettger Y, Kromminga A. Managing unwanted immunogenicity of biologicals. Autoimmun Rev. 2015;14(7):569-74.

3. Ogrič M, Terčelj M, Praprotnik S, et al. Detection of adalimumab and anti-adalimumab antibodies in patients with rheumatoid arthritis-a comprehensive overview of methodology pitfalls and benefits. Immunol Res. 2016. doi:10.1007/s12026-016-8824-8.

4. Audemard-Verger A, Pillebout E, Guillevin L, Thervet E, Terrier B. IgA vasculitis (Henoch-Shönlein purpura) in adults: diagnostic and therapeutic aspects. Autoimmun Rev. 2015;14(7):579-85.

5. Fenoglio R, Naretto C, Basolo B, et al. Rituximab therapy for IgA-vasculitis with nephritis: A case series and review of the literature. Immunol Res. 2016. doi:10.1007/s12026-016-8827-5.

6. Katchan V, David P, Shoenfeld Y. A 47-year old woman with idiopathic thrombocytopenic purpura and polyneuropathy. Immunol Res. 2016. doi:10.1007/s12026-016-8828-4.

7. Wu H, Zhao M, Tan L, Lu Q. The key culprit in the pathogenesis of systemic lupus erythematosus: aberrant DNA methylation. Autoimmun Rev. 2016;15(7):684-9.

8. Marinho A, Carvalho C, Boleixa D, et al. Vitamin D supplementation effects on FoxP3 expression in T cells and FoxP3 ${ }^{+} / \mathrm{IL}-$ $17 \mathrm{~A}$ ratio and clinical course in systemic lupus erythematosus 
patients: a study in a Portuguese cohort. Immunol Res. 2016. doi:10.1007/s12026-016-8829-3.

9. Rizzo MI, Greco A, De Virgilio A, et al. Glaucoma recent advances in the involvement of autoimmunity. Immunol Res. 2016. doi:10.1007/s12026-016-8837-3.

10. Goulabchand R, Hafidi A, Millet I, et al. Mastitis associated with Sjögren's syndrome: a series of nine cases. Immunol Res. 2016. doi:10.1007/s12026-016-8830-x.

11. Abreu MM, Danowski A, Wahl DG, Amigo MC, Tektonidou M, Pacheco MS, et al. The relevance of "non-criteria" clinical manifestations of antiphospholipid syndrome: 14th International Congress on antiphospholipid antibodies technical task force report on antiphospholipid syndrome clinical features. Autoimmun Rev. 2015;14(5):401-14.

12. Hassan M. Noureldine A, Haydar AA, et al. Antiphospholipid syndrome (aps) revisited: would migraine headaches be included in future classification criteria? Immunol Res. 2016.. doi:10.1007/ s12026-016-8831-9.

13. Kahaly GJ, Hansen MP. Type 1 diabetes associated autoimmunity. Autoimmun Rev. 2016;15(7):644-8.

14. Gianchecchi E, Fierabracci A. On the pathogenesis of insulindependent diabetes mellitus: the role of microbiota. Immunol Res. 2016. doi:10.1007/s12026-016-8832-8.

15. Patuzzo G, Barbieri A, Tinazzi E, Veneri D, Argentino G, Moretta F, Puccetti A, Lunardi C. Autoimmunity and infection in common variable immunodeficiency (CVID). Autoimmun Rev. 2016;15:877-82.

16. Gendelman O, Mahroum N, Comaneshter D, et al. Hepatitis B carrier state among SLE patients: case-control study. Immunol Res. 2016. doi:10.1007/s12026-016-8834-6.
17. Shovman O, Gilburd B, Chayat C, et al. Anti-HMGCR antibodies demonstrate high diagnostic value in the diagnosis of immune mediated necrotizing myopathy following statin exposure. Immunol Res. 2016. doi:10.1007/s12026-016-8867-x.

18. Pellegrino P, Clementi E, Radice S. On vaccine's adjuvants and autoimmunity: current evidence and future perspectives. Autoimmun Rev. 2015;14(10):880-8.

19. Blitshteyn S, Brook J. Postural tachycardia syndrome (POTS) with anti-NMDA receptor antibodies after human papillomavirus vaccination. Immunol Res. 2016. doi:10.1007/s12026-016-88551.

20. Balbi GGM, Montes RA, Vilela VS, et al. Rapidly progressive diffuse systemic sclerosis after local vitamins A, D and E complex injections: literature review and report of two cases. Immunol Res. 2016. doi:10.1007/s12026-016-8851-5.

21. Valentini G. Undifferentiated Connective tissue disease at risk for systemic sclerosis (SSc) (so far referred to as very early/early SSc or pre-SSc). Autoimmun Rev. 2015;14(3):210-3.

22. Infantino M, Meacci F, Grossi V, et al. The clinical impact of anti-DFS70 antibodies in undifferentiated connective tissue disease: case reports and a review of the literature. Immunol Res. 2016. doi:10.1007/s12026-016-8836-4.

23. Houri Levi E, Watad A, Whitby A, Tiosano S, Comaneshter D, Cohen AD, Amital H. Coexistence of ischemic heart disease and rheumatoid arthritis patients-a case control study. Autoimmun Rev. 2016;15(4):393-6.

24. Lazzerini PE, Capecchi PL, Galeazzi M, Laghi-Pasini F. Biologic drugs and arrhythmic risk in chronic inflammatory arthritis: the good and the bad. Immunol Res. 2016. doi:10.1007/s12026-0168833-7. 